CERN-EP/2001-074

12th October 2001

\title{
The large area Emulsion Tracker of the CHORUS experiment
}

\author{
S. Aoki ${ }^{1)}$, J. Dupraz ${ }^{2)}$, J.P. Fabre ${ }^{2)}$, W. Flegel ${ }^{2)}$, T. Hara ${ }^{1)}$, Y. Ishii ${ }^{3)}$, T. Kawamura ${ }^{3, a)}$, \\ D.C. Kim ${ }^{4)}$, T. Kurihara ${ }^{3)}$, E. Niu ${ }^{2, b)}$, S. Ogawa ${ }^{3)}$, H. Shibuya ${ }^{3, *)}$, J.S. Song ${ }^{4}$, \\ G. Van Beek ${ }^{5)}$, P. Vilain ${ }^{5)}$, J. Watanabe ${ }^{3)}$, G. Wilquet ${ }^{5)}$, C.S. Yoon ${ }^{4)}$
}

\begin{abstract}
A new type of tracking device using large area nuclear emulsion sheets was developed for the short baseline neutrino oscillation experiment, CERN-WA95/CHORUS. The emulsion tracker is made of three layers, each $25 \mathrm{~mm}$ thick including support, and covering an area of about $5 \mathrm{~m}^{2}$. Two layers were installed upstream and one downstream of the hexagonal magnet of the CHORUS hadron spectrometer. The achieved position resolution over the whole area is $25 \mu \mathrm{m}$, which keeps the momentum resolution of the spectrometer below $30 \%$ up to momenta of about $15 \mathrm{GeV} / c$.
\end{abstract}

(To be submitted to NIM.)

\footnotetext{
1) Kobe University, Kobe, Japan

2) CERN, Geneva, Switzerland

3) Toho University, Funabashi, Japan

4) Gyeongsang National University, Jinju, South Korea

5) Inter-University Institute for High Energies (ULB-VUB) Brussels

a) Now at Università Federico II and INFN, Naples, Italy.

b) Now at Center for Chronological Research, Nagoya University, Nagoya, Japan.

*) Corresponding author: shibuya@ph.sci.toho-u.ac.jp
} 
The nuclear emulsion has the best spatial resolution among all detectors currently used in high energy physics and is thus well suited for observing directly the production and decay of short-lived particles. It has often been used as an active target in hybrid experiments $[1,2,3,4,5]$. However, the low scanning speed of the microscope system has until recently limited the practical use of the information recorded in the emulsion.

The remarkable increase in scanning speed achieved nowadays by automatic scanning techniques makes it possible not only to extend the use of nuclear emulsion to very large volumes $[6,7]$ and then to improve the sensitivity of this kind of experiments $[8,9,10]$, but also to widen its range of application as detector element.

A large and precise tracking device has been developed for the CHORUS hadron spectrometer [6]. This paper describes its aim, structure, data taking, alignment and performance. We describe in some detail the alignment procedure, which is a key issue in order to obtain a good spatial resolution over a large area.

\section{The Emulsion Tracker System \\ 2.1 Aim}

A schematic view of the CHORUS detector is shown in Figure 1. The experimental setup is composed of an emulsion target, a scintillating fiber tracker system, scintillator trigger hodoscopes, a hadron spectrometer, a lead/scintillator calorimeter and a muon spectrometer. Detailed descriptions of the apparatus are given elsewhere [6]. The CHORUS experiment searches for neutrino oscillation in the $\nu_{\mu} \rightarrow \nu_{\tau}$ appearance channel. The occurrence of the reaction $\nu_{\tau} N \rightarrow \tau^{-} X$ and the subsequent decay of the short-lived $\tau^{-}$ are directly detected in the emulsion target [7]. Hadronic decay modes of the $\tau^{-}$lepton have a large branching fraction, about $65 \%$, dominantly into a single charged prong. It is thus important to identify hadronic decays in order to increase the detection efficiency of the $\tau^{-}$lepton. However there are sources of background such as strange or charm particle decays and secondary interactions, which mimic the $\tau^{-}$hadronic decays. This background is effectively rejected by a transverse momentum cut with respect to the parent particle direction and the requirement of negative charge. It is therefore very important to measure hadron momenta accurately.

The CHORUS hadron spectrometer consisted initially of three diamond-shaped scintillating fiber trackers [11], one upstream(DT1) and two downstream(DT2 and DT3) of the air-core hexagonal magnet [12], as shown in Figure 2. The magnet produces a homogeneous field of $0.118 \mathrm{~T}$. Thanks to the special design of the magnet, the field lines are parallel to the outer sides of the hexagon and the magnetised region extends for a depth of $0.75 \mathrm{~m}$ in the direction of the beam.

Three layers of large area Emulsion Trackers, labelled ET0, ET1 and ET2, were installed for the 1996 and 1997 runs $^{1)}$ as a detector upgrade [13, 14]. The aim was to improve the accuracy of the momentum measurement and to extend the recognition of the hadron charge from $10 \mathrm{GeV} / c$ to $30 \mathrm{GeV} / c$. This kinematical domain includes an additional $26 \%$ of the $\tau \rightarrow h^{-} X$ decays, as shown in Figure 3.

\subsection{Design of the Emulsion Tracker system}

For each selected track, the trajectory reconstructed in the scintillating fiber tracker system is used to define, in each layer, the area in which the corresponding track segment

\footnotetext{
1) Just before starting the 1995 run, only ET1 and ET2 were installed to test the tracking capability.
} The alignment and scanning procedures were established through this test exposure. 
will be searched. For ET1 and ET2, the predicted area is minimized by installing these trackers in the small gaps between the DT's and the magnet (50 and $30 \mathrm{~mm}$ for DT1 and DT2 respectively). ET0 was installed at the most upstream available position.

ET0 is composed of 8 juxtaposed emulsion sheets, while both ET1 and ET2 consist of 18 sheets. Each sheet has an emulsion layer of $100 \mu \mathrm{m}$ on each side of a $800 \mu \mathrm{m}$ thick $0.74 \times 0.37 \mathrm{~m}^{2}$ acrylic support. The emulsion sheets are wrapped with a laminated sheet and packed in vacuum. The package is then attached by vacuum to a $20 \mathrm{~mm}$ thick honeycomb board. The total thickness is about $25 \mathrm{~mm}$. The honeycomb boards of ET1 and ET2 were firmly fixed to the supports of DT1 and DT2 respectively, while the honeycomb board of ET0 was fixed to the frame of trigger counters just downstream of the target fiber tracker.

To reduce the fading of tracks recorded in the emulsion, the target zone was kept at a temperature of $(5.0 \pm 0.5)^{\circ} \mathrm{C}$ in a cool box including the hexagonal magnet and the fiber trackers. As a result, the change in the lateral dimensions of the ET emulsion sheets due to thermal variations was minimized.

Possible effects from the heat of the hexagonal magnet and from vibration due to the magnet pulsing were also considered. To minimize the vibration effects, strong support boards were needed for ET1 and ET2. To protect the emulsion sheets from the heat of the magnet, heat insulation curtains were introduced between the magnet and the emulsion packages of ET1 and ET2. Each curtain is composed of a plastic bubble sheet and a socalled "super-insulation" sheet made of 10 thin sheets of aluminized mylar. The curtain is fixed to the magnet by a net so that cold air can pass in between. Two thermometers were set to monitor the surface temperature of the emulsion sheets. One is attached to the ET1 honeycomb board $1 \mathrm{~m}$ below the top, the other to the ET2 $1 \mathrm{~m}$ above the bottom.

The amount of material traversed by particles had to be minimized to avoid multiple scattering and secondary interactions. The total material of each ET layer is $1.3 \%$ of a radiation length. The amount of material of the Emulsion Tracker and the other components of the hadron spectrometer is summarized in Table 1.

The distance along the beam direction from the most downstream emulsion of the main target to ET0 is $\sim 0.26 \mathrm{~m}$, the distance between ET0 and ET1 is $\sim 0.43 \mathrm{~m}$ and the distance between ET1 and ET2 is $\sim 0.96 \mathrm{~m}$.

To know the position of each sheet relative to the honeycomb board, X-ray guns, shown in Figure 4, were used. Each X-ray gun is made of a brass cylinder filled with ${ }^{55} \mathrm{Fe}$ source. The X-ray gun produces a black spot of $1 \mathrm{~mm}$ diameter in the emulsion sheet. A set of 18 X-ray guns per emulsion sheet was placed in holes drilled in the honeycomb board. The arrangement of the emulsion sheets and the positions of the X-ray guns are shown in Figures 5 and 6.

\subsection{Survey and position monitoring}

The relative three-dimensional positions of the 144(324) holes for X-ray guns on ET0(ET1,2) honeycomb board were measured prior to installation by using the digital photogrammetry method [15] at room temperature, $\sim 20^{\circ} \mathrm{C}$. Several photogrammetric images of each honeycomb board were taken by a digital camera from different directions. The images were processed and the three dimensional relative positions were reconstructed..$^{2)}$ The spatial resolution is $20 \mu \mathrm{m}$ in the transverse direction and $30 \mu \mathrm{m}$ in depth

2) Camera: NIKON focal length $28 \mathrm{~mm}$, CCD: Kodak DCS $4603000 \times 2000$ pixels, data taking and image processing software: an Imetric product. 
over the whole board. After installing the honeycomb boards in the CHORUS apparatus, the absolute positions of 18 holes on ET1 and ET2 honeycomb boards and aluminum columns attached at the side of each board were measured by using a theodolite at $5^{\circ} \mathrm{C}$ in the cool box. The final positions of all holes were then deduced by means of a geometrical adjustment.

Furthermore, in order to monitor relative movements of some detector elements, four RASNIK(Relative Alignment System of NIKHEF) optical alignment systems [16] were installed in 1996. The RASNIK system consists of a light source with a "coded mask", a lens and a CCD camera. If any of the three elements moves, a corresponding movement of the image is observed. One of the RASNIK systems was mounted on DT1 while three other systems were mounted on chambers newly installed for a CHORUS upgrade, the Honeycomb Trackers [6]. Since the ET1 honeycomb board was fixed to the DT1, relative movement of ET1 during the data taking period is also monitored by the DT1 RASNIK.

\subsection{Installation and data taking}

To keep the rate of background tracks as low as possible, the emulsions were poured just before installation and the sheets were developed at the CERN emulsion facility just after removal.

The ET emulsion sheets had to be replaced in the middle of each year's data taking to limit the background due to passing through tracks. The support honeycomb boards were first installed in the CHORUS apparatus from the top by opening the roof of the cool box at the beginning of the 1996 run. The emulsion sheets were inserted from the top of the apparatus into the corresponding narrow gaps by folding the packages without opening the roof, as shown in Figure 7, in order to keep the temperature stable.

After installation of the ETs, the emulsion target has been exposed to the neutrino beam in the 1996 and 1997 runs, $3.05 \times 10^{19}$ protons on target (PoT) in total. Each run lasted six months and all the ET emulsion sheets were changed every three months. Thus, the ET exposure in the 1996 and 1997 runs was divided into four periods. For each period, special data from beam associated muons passing through the detector while the magnet was switched off were taken to align the emulsion sheets with respect to the whole detector.

\section{$3 \quad$ Scanning and Analysis Procedures}

\subsection{Scanning}

Tracks reconstructed in the fiber tracking system are interpolated to the ET layers. Their positions and slopes are used to search for the corresponding ET track segments. The typical scanning area is about $1 \mathrm{~mm}^{2}$, as determined from the fiber tracking resolution. Scanning is performed by fully automatic microscope systems [17].

The fiber prediction coordinates are expressed in the general CHORUS reference frame $^{3)}$ while the measurements in emulsion are performed with respect to the X-ray marks. The position of these reference marks may be affected by the emulsion sheet expansion at the temperature of the scanning room. For this reason, the $9 \mathrm{X}$-ray mark coordinates $\left(Y_{i}, Z_{i}\right)$ of each half sheet are measured at the beginning of each scanning

3) A common coordinate system is used for the description of CHORUS detector geometry. It is a righthanded cartesian system in which $X$ axis is the horizontal projection of the neutrino beam axis, $Y$ and $Z$ are the horizontal and upward vertical transverse axes respectively. 
session. Their coordinates $\left(Y_{i}^{\prime}, Z_{i}^{\prime}\right)$ are then computed in the CHORUS frame using an affine transformation:

$$
\left(\begin{array}{c}
Y_{i}^{\prime} \\
Z_{i}^{\prime}
\end{array}\right)=\left(\begin{array}{ll}
a & b \\
c & d
\end{array}\right)\left(\begin{array}{c}
Y_{i} \\
Z_{i}
\end{array}\right)+\left(\begin{array}{c}
p \\
q
\end{array}\right) .
$$

where the parameters $a, b, c, d, p, q$ are obtained by a $\chi^{2}$ minimization using as constraint the surveyed positions of the X-ray guns. Within the predicted scanning area, track segments with a direction compatible with the one defined by the fiber tracker are then searched. The track finding efficiency of the automatic scanning system was measured to be greater than $98 \%$ for tracks with slope less than 0.4, as shown in Figure 8.

\subsection{Alignment of the Emulsion Tracker relative to the fiber tracker $\operatorname{system}(\mathrm{ET}-\mathrm{DT})$}

One expects some systematic uncertainties on the three dimensional positions and slopes due, for instance, to the non perfect planarity of the honeycomb boards and to shrinkage or expansion of the emulsion sheets. A specific alignment of each ET half sheet to the fiber tracker system was performed to achieve a better matching between the fiber predictions and the emulsion measurements. Both beam associated muons (with the magnet off) and high momentum muons from $\nu_{\mu}$ charged current interactions (with the magnet on) were used for this purpose.

After measurement of a few hundreds of muon tracks per half emulsion sheet, the corrections on the track segment positions $\left(Y_{i}, Z_{i}\right)$ and slopes $\left(A y_{i}, A z_{i}\right)$ were obtained, for each half sheet, by minimizing the following quantities:

$$
\begin{aligned}
S_{\text {position }} & =\sum_{i=1}^{n}\left\{\left(Y_{i}^{\prime}-Y_{\mathrm{p} i}\right)^{2}+\left(Z_{i}^{\prime}-Z_{\mathrm{p} i}\right)^{2}\right\}, \\
S_{\text {slope }} & =\sum_{i=1}^{n}\left\{\left(A y_{i}^{\prime}-A y_{\mathrm{p} i}\right)^{2}+\left(A z_{i}^{\prime}-A z_{\mathrm{p} i}\right)^{2}\right\},
\end{aligned}
$$

where $Y_{\mathrm{p} i}$ and $Z_{\mathrm{p} i}$ are the predicted coordinates, $A y_{\mathrm{p} i}$ and $A z_{\mathrm{p} i}$ the predicted slopes, and

$$
\begin{aligned}
\left(\begin{array}{c}
Y_{i}^{\prime} \\
Z_{i}^{\prime}
\end{array}\right) & =\left(\begin{array}{cc}
a & b \\
c & d
\end{array}\right)\left(\begin{array}{c}
Y_{i}+A y_{i} \cdot x_{\mathrm{s}} \\
Z_{i}+A z_{i} \cdot x_{\mathrm{s}}
\end{array}\right)+\left(\begin{array}{c}
p \\
q
\end{array}\right), \\
\left(\begin{array}{c}
A y_{i}^{\prime} \\
A z_{i}^{\prime}
\end{array}\right) & =\left(\begin{array}{c}
A y_{i} \\
A z_{i}
\end{array}\right)+\left(\begin{array}{c}
a y_{\mathrm{s}} \\
a z_{\mathrm{s}}
\end{array}\right),
\end{aligned}
$$

$x_{\mathrm{s}}$ is a shift parameter along the $X$ axis, $a y_{\mathrm{s}}$ and $a z_{\mathrm{s}}$ are slope shift parameters, $a, b, c, d, p$ and $q$ are the parameters of an affine transformation.

\subsection{Alignment among the sheets of the Emulsion Tracker system(ET-ET)}

The alignment described in the previous section mainly aims at improving the track finding efficiency by a better matching to the fiber tracker predictions. In order to obtain from the Emulsion Tracker an independent momentum determination, a last alignment step is performed by using beam associated muons with the magnet off, crossing all the three planes. 
The track segments measured in ET0 and ET1 are used to define a line of slopes $\left(A y_{i}, A z_{i}\right)$ which extrapolates to the position $\left(Y_{\text {ext } i}, Z_{\text {ext } i}\right)$ at the ET2 plane. The ET2 plane is then aligned by minimizing the quantity:

$$
S_{\text {position }}=\sum_{i=1}^{n}\left\{\left(Y_{\mathrm{ET} 2 i}^{\prime}-Y_{\mathrm{ext} i}\right)^{2}+\left(Z_{\mathrm{ET} 2 i}^{\prime}-Z_{\mathrm{ext} i}\right)^{2}\right\},
$$

where

$$
\left(\begin{array}{c}
Y_{\mathrm{ET} 2 i}^{\prime} \\
Z_{\mathrm{ET} 2 i}^{\prime}
\end{array}\right)=\left(\begin{array}{cc}
a & b \\
c & d
\end{array}\right)\left(\begin{array}{c}
Y_{\mathrm{ET} 2 i}+A y_{i} \cdot x_{\mathrm{s}} \\
Z_{\mathrm{ET} 2 i}+A z_{i} \cdot x_{\mathrm{s}}
\end{array}\right)+\left(\begin{array}{c}
p \\
q
\end{array}\right),
$$

$Y_{\mathrm{ET} 2 i}$ and $Z_{\mathrm{ET} 2 i}$ are the track segment positions measured in ET2. The parameters of the affine transformation were determined separately for each set of 3 half-sheets sharing common tracks.

\subsection{Momentum measurement with the Emulsion Trackers}

The displacement between the intercepts at ET2 of trajectories computed with and without the magnetic deflection gives a measure of the momentum. The hexagonal magnet consists of six equilateral triangles and the direction of the magnetic field is parallel to the outer sides of the triangles.

In the approximation of a small bending angle, the displacement $d[\mathrm{~m}]$ in the bending plane, shown in Figure 9, can be expressed as:

$$
\begin{aligned}
d & =\Delta \theta \cdot D \cdot\left(1+\tan ^{2} \theta\right) \\
& =0.3 \cdot \frac{L \cdot B}{p} \cdot D \cdot\left(1+\tan ^{2} \theta\right)^{\frac{3}{2}}
\end{aligned}
$$

where $D[\mathrm{~m}]$ is the distance between the magnet center and ET2, $L[\mathrm{~m}]$ the length of the magnet, $B[\mathrm{~T}]$ the magnetic field, $\Delta \theta$ the bending angle, $\theta$ the angle with respect to the $X$ axis and $p[\mathrm{GeV} / c]$ the projection of the momentum in the bending plane. Substituting $D=0.48 \mathrm{~m}, L=0.75 \mathrm{~m}$ and $B=0.118 \mathrm{~T}$, the displacement $d[\mathrm{~m}]$ can be written as:

$$
d \sim \frac{0.0127}{p} \cdot\left(1+\tan ^{2} \theta\right)^{\frac{3}{2}}
$$

In practice, the momentum determination is slightly more complicated since, as shown in Figure 9, both ends of the magnetized volume are arc-shaped and the effective magnet length $L$ needs to be evaluated for each trajectory.

\section{$4 \quad$ Results and Detector Performance}

\subsection{Application of the alignment procedures}

The method presented in Section 3.2 and 3.3 can be applied only after a sufficient number of muon tracks have been located in the emulsion sheets. To collect this first sample, a coarse alignment of each ET layer was performed on the basis of the geometrical survey of the X-ray guns and the monitoring data of the RASNIK system. 
The RASNIK system, mounted on DT1, observed about $1 \mathrm{~mm}$ movement of the DT1 center along the beam direction, which coincides with the temperature change of about $5{ }^{\circ} \mathrm{C}$ whenever the hexagonal magnet is switched on (or off) between alignment and neutrino data taking runs. The measured movement closely follows the temperature evolution which has a transition time of the order of one hour. It was also found that the displacement of DT1 along the beam was roughly varying linearly with the distance from the center while the edges were fixed. The magnet temperature was permanently monitored and the DT1 displacements have only a marginal effect if the data used for alignment correspond to periods of stable temperature conditions.

The survey results indicate a maximum deviation from planarity of the Emulsion Trackers of a few mm. For the coarse alignment, a simple plane equation was fitted to the positions of the X-ray guns in a full ET layer. The final alignments using muon tracks were performed on a half sheet basis to correct for possible deformation of the ET's.

The results of the ET-DT alignment are summarized in Table 2. The R.M.S. deviations in position and slope of the tracks found in the emulsion from the fiber predictions are given separately for beam muons recorded with the magnet off and for muons from neutrino interactions (magnet on). These values merely reflect the accuracy of the reconstruction by the fiber trackers.

After the ET-ET alignment, the deviation of the tracks found in ET2 from their expected position using the tracks in ET0 and ET1 has a gaussian shape with $\sigma \sim 100 \mu \mathrm{m}$, as shown in Figure 10. Under the assumption that the measurement errors are identical and uncorrelated, such a residual value would correspond to a position measurement error in each layer of $\sigma \sim 25 \mu \mathrm{m}$.

\subsection{Momentum resolution}

The momentum resolution of the ET system was evaluated by comparing the muon momentum values measured both in the ET's and in the downstream muon spectrometer (after correction of the energy loss in the calorimeter). The comparison is illustrated in Figure 11. The muon spectrometer system has a resolution of $\Delta p / p \sim 7 \%$ below $6 \mathrm{GeV} / c$ and $\sim 16.5 \%$ above $6 \mathrm{GeV} / c[6]$.

Figure 12 shows the R.M.S. values of the relative difference between the measurements in the muon spectrometer and in the ET as a function of the muon spectrometer momentum. This distribution is well reproduced by convoluting the above spectrometer resolution with a momentum dependent term given by $\Delta p / p \sim 1.0 \% \cdot p[\mathrm{GeV} / c]$ and with a constant term of $22 \%$ due to the multiple scattering in the traversed materials, as indicated by the dashed line in Figure 12.

\subsection{Background density}

In emulsion, all tracks accumulated between the sheet production and its development contribute to the background. The background density was estimated by scanning large areas distributed over the different sheets. Figure 13 shows the slope distribution of the background tracks. Three peaks, $(A y, A z)_{\text {center }} \sim(0.00,0.04),(-0.08,-0.01)$, $(-0.13,-0.01)$ are seen. The first peak corresponds to the neutrino beam direction and the latter two correspond to directions of muons originating from test beams in the West Hall. The background density was measured to be $\sim 40$ tracks $/ \mathrm{mm}^{2}$ for tracks with slopes $<0.3$. In a typical scanning area of $1 \mathrm{~mm}^{2}$, the rate of background tracks which match the predicted track slopes within a tolerance of 0.003 is up to $7 \times 10^{-2}$ for directions close to the neutrino beam and less than $10^{-2}$ for larger slopes, as shown in Figure 14. 
The achieved momentum resolution shown in Figure 12 corresponds to an effective position error of $32 \mu \mathrm{m}$ on each ET layer, slightly larger than the value of $25 \mu \mathrm{m}$ obtained with the magnet-off alignment muon data. The difference might be due to a residual temperature effect of $\sim 10 \mu \mathrm{m}$ in spite of the presence of the heat insulation curtain. Even a resolution of $25 \mu \mathrm{m}$ is substantially larger than the intrinsic accuracy of the microscope and could still be improved by further local alignment. However, the present momentum resolution is sufficient to satisfy the CHORUS requirements, given the energy spectrum of the $\tau$-decay products. It may also be noted that the installation of the ET's hardly affect the large constant term of $22 \%$ in the momentum resolution which is almost entirely due to the magnet and fiber tracker materials (see Table 1).

Up to now, the CHORUS analysis $[8,9,10]$ has been based on an event selection using only the fiber tracker information. In a second phase of the analysis, the neutrino interactions will be rescanned with the so-called Net-Scan technique [18] in order to establish the full event topology in the vertex region. All the observed decay tracks, including those not previously reconstructed in the fiber tracker system or with unknown momentum, will be followed down to the downstream trackers. Whenever possible, they will be searched in the ET layers and momentum analyzed. The better momentum resolution of the ET system significantly improves the background rejection power and thus the sensitivity of the experiment to the neutrino oscillation, as anticipated in Section 2.1.

\section{Conclusions}

A large area Emulsion Tracker system has been successfully constructed and installed in the CHORUS apparatus at the beginning of the 1996 run. The CHORUS data taking with the ET system was carried out until the end of the experiment, in November 1997. A careful ET alignment allowed the scanning based on the fiber tracker predictions. The position resolution was obtained to be as small as $25 \mu \mathrm{m}$ over an area of $2.22 \times 2.22 \mathrm{~m}^{2}$. A momentum resolution of $\Delta p / p=\sqrt{(0.22)^{2}+(0.010 \cdot p[\mathrm{GeV} / c])^{2}}$ was obtained after the alignment among the ETs. The ET system showed a good performance as a large area tracking device.

\section{Acknowledgements}

The authors would like to thank the colleagues in the CHORUS collaboration for their continuous support and assistance. We are also indebted to the SPS staff for the efficient accelerator operation, to L. Gatignon for the West Area test beams, and to C. Lasseur and the CERN survey group. We gratefully acknowledge the financial support of the different funding agencies: in particular, the Promotion and Mutual Aid Corporation for Private Schools of Japan, Japan Society for the Promotion of Science (Japan); and the Korea Research Foundation (Grant KRF-99-005-D00004) (Republic of Korea); the Institut Interuniversitaire des Sciences Nucléaires and the Interuniversitair Instituut voor Kernwetenschappen (Belgium). 


\section{References}

[1] E.H.S. Burhop et al., Phys. Lett. 65B (1976) 299.

[2] D. Allasia et al., WA17 Collaboration, Nucl. Phys. B 176 (1980) 13.

[3] N. Ushida et al., E531 Collaboration, Nucl. Instr. and Meth. 224 (1984) 50.

[4] S. Aoki et al., WA75 Collaboration, Nucl. Instr. and Meth. A 274 (1989) 64.

[5] K. Kodama et al., E653 Collaboration, Nucl. Instr. and Meth. A 289 (1990) 146.

[6] E. Eskut et al., CHORUS Collaboration, Nucl. Instr. and Meth. A 401 (1997) 7.

[7] S. Aoki et al., CHORUS Collaboration, Nucl. Instr. and Meth. A 447 (2000) 361.

[8] E. Eskut et al., CHORUS Collaboration, Phys. Lett. B 424 (1998) 202.

[9] E. Eskut et al., CHORUS Collaboration, Phys. Lett. B 434 (1998) 205.

[10] E. Eskut et al., CHORUS Collaboration, Phys. Lett. B 497 (2001) 8.

[11] P. Annis et al., Nucl. Instr. and Meth. A 412 (1998) 19.

[12] F. Bergsma et al., Nucl. Instr. and Meth. A 357 (1995) 243.

[13] CHORUS Collaboration, CERN-SPSLC/96-4, SPSLC/M571 (1996).

[14] T. Kawamura, Ph.D. Thesis, Toho University, 1999.

[15] C. Lasseur and the CERN survey group, http://www.cern.ch/SurvExpDiffusion/chorus.html

[16] J. Dupraz, D. Saltzberg and G. Van Beek, Nucl. Instr. and Meth. A 388 (1997) 173.

[17] T. Nakano, Ph.D. Thesis, Nagoya University, 1997.

[18] S. Aoki, in: Proc. 9th Int. Workshop on Vertex Detectors, Michigan 2000, to be published in Nucl. Instr. and Meth. A. 


\section{CHORUS}

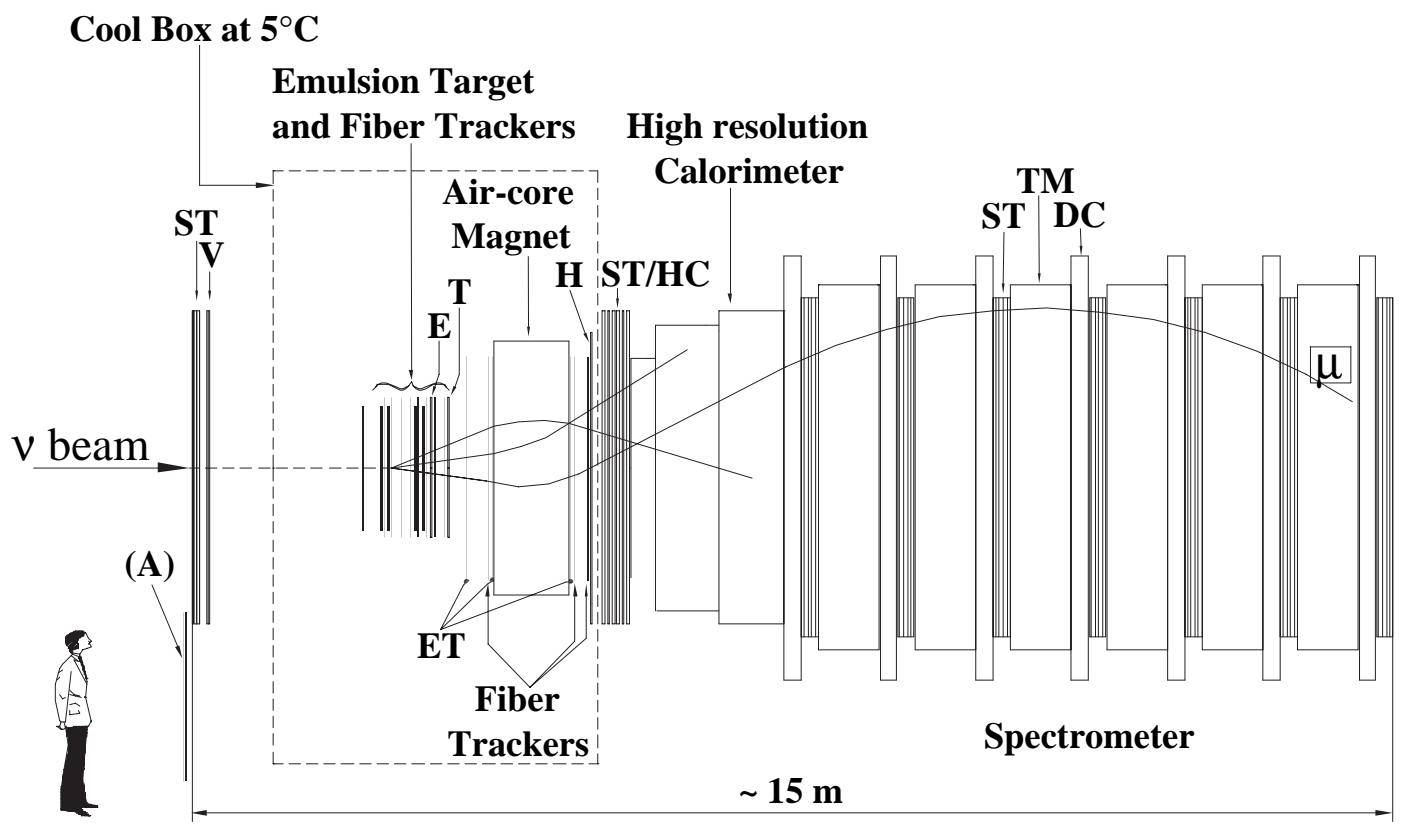

Figure 1: Schematic diagram (side view) of the CHORUS apparatus. V,A,T,E and H are trigger and veto hodoscopes. ST, TM and DC are streamer tubes, iron toroidal magnets and drift chambers, respectively, of the muon spectrometer system. The detector upgrades in 1996 on the emulsion trackers and honeycomb chambers are denoted by ET and HC, respectively. 


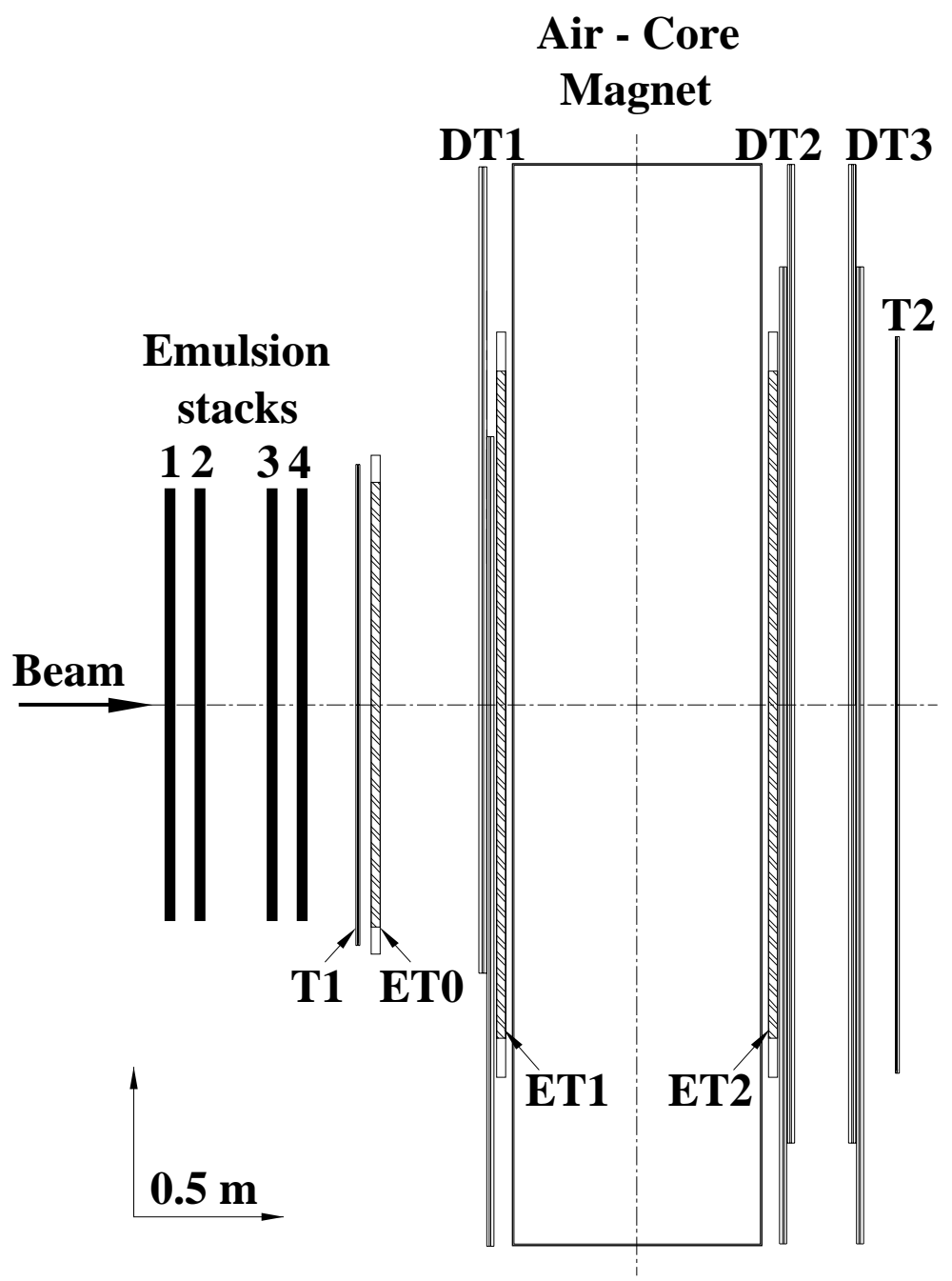

Figure 2: The geometry of the hexagonal air-core magnet and the diamond shaped magnet trackers (DT1, DT2, DT3). The emulsion trackers (ET0, ET1, ET2) are also shown. 


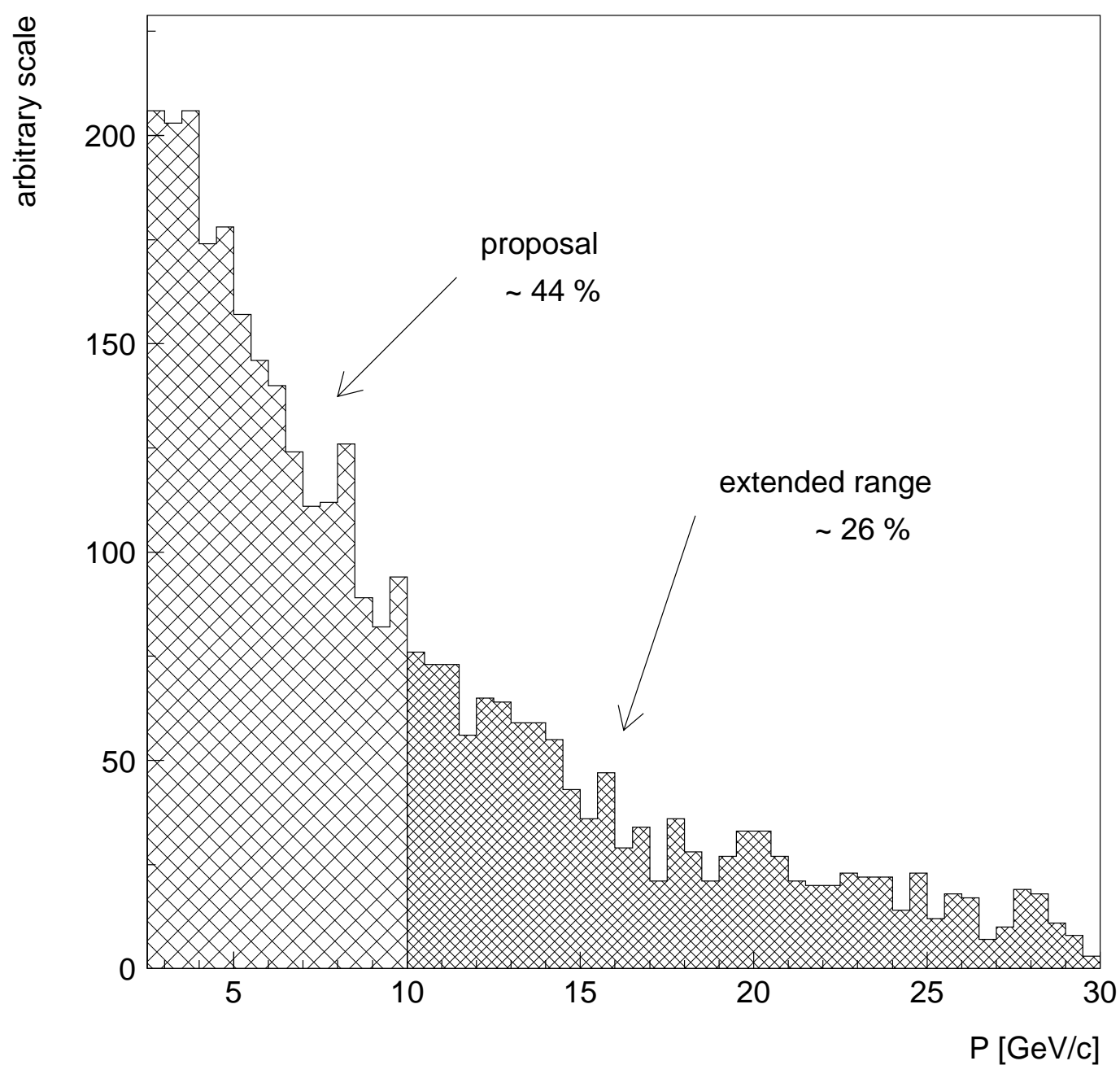

Figure 3: Momentum distribution of negative hadrons from one-prong $\tau^{-}$decays. 


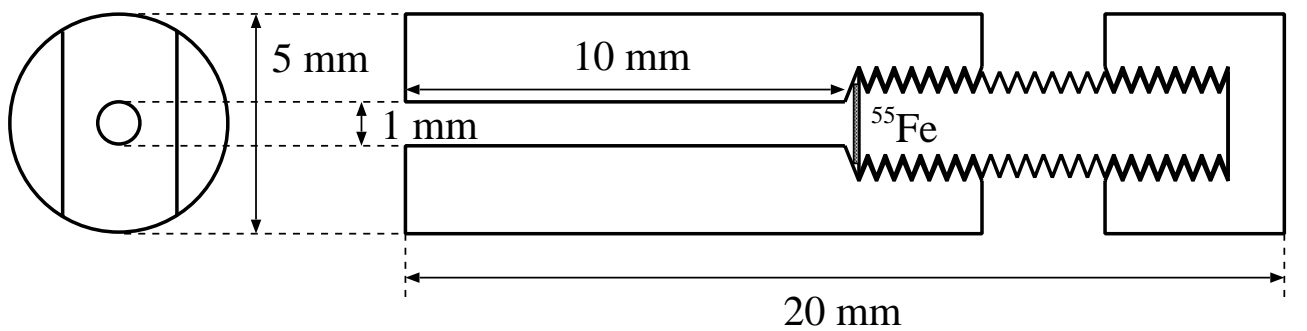

Figure 4: Cross section of an X-ray gun. The collimator length is adjusted at $10 \mathrm{~mm}$. 


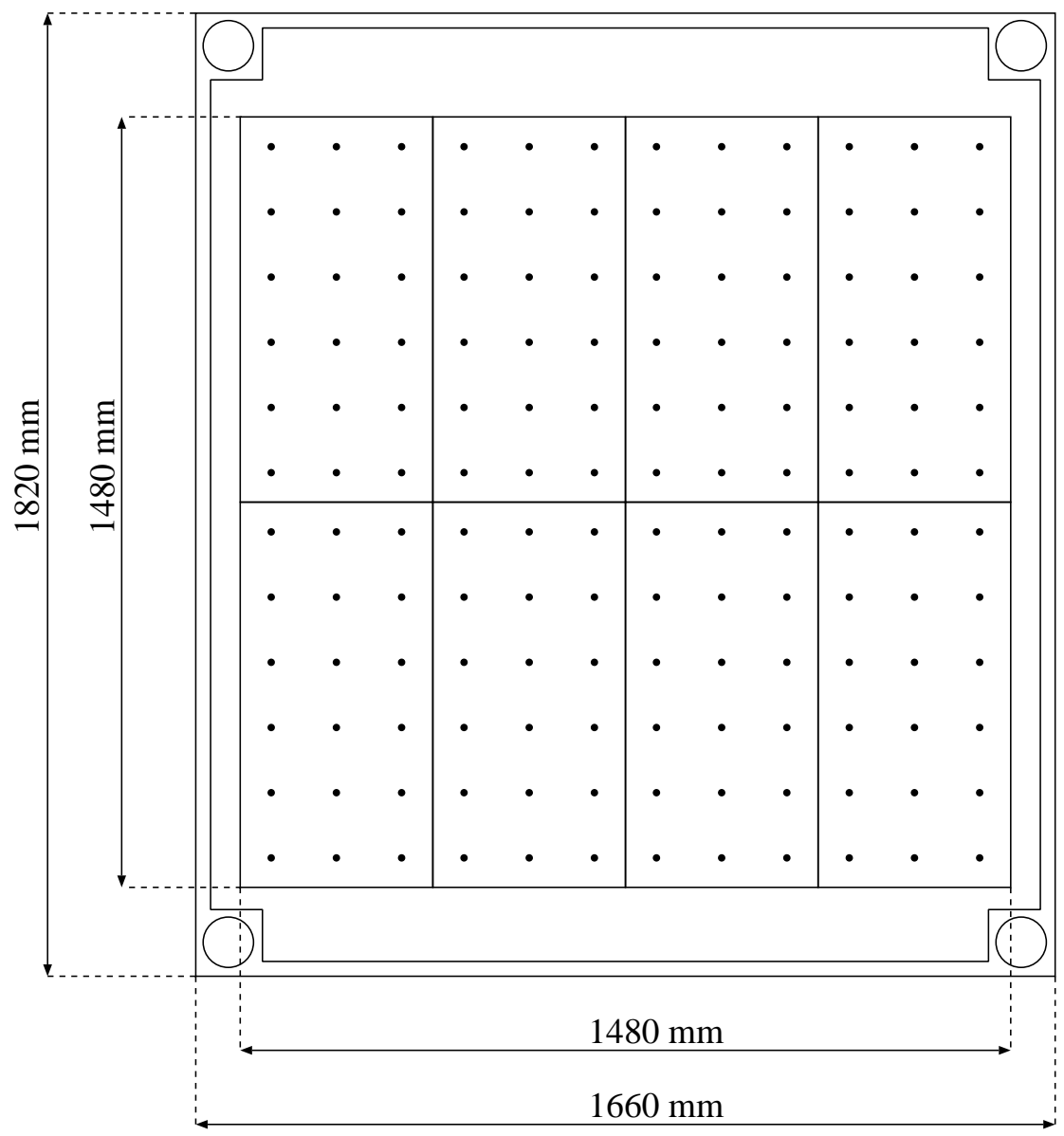

Figure 5: Front view of ET0, showing the arrangement of eight emulsion plates. The positions of the X-ray guns embedded in the honeycomb board are shown by black dots. 


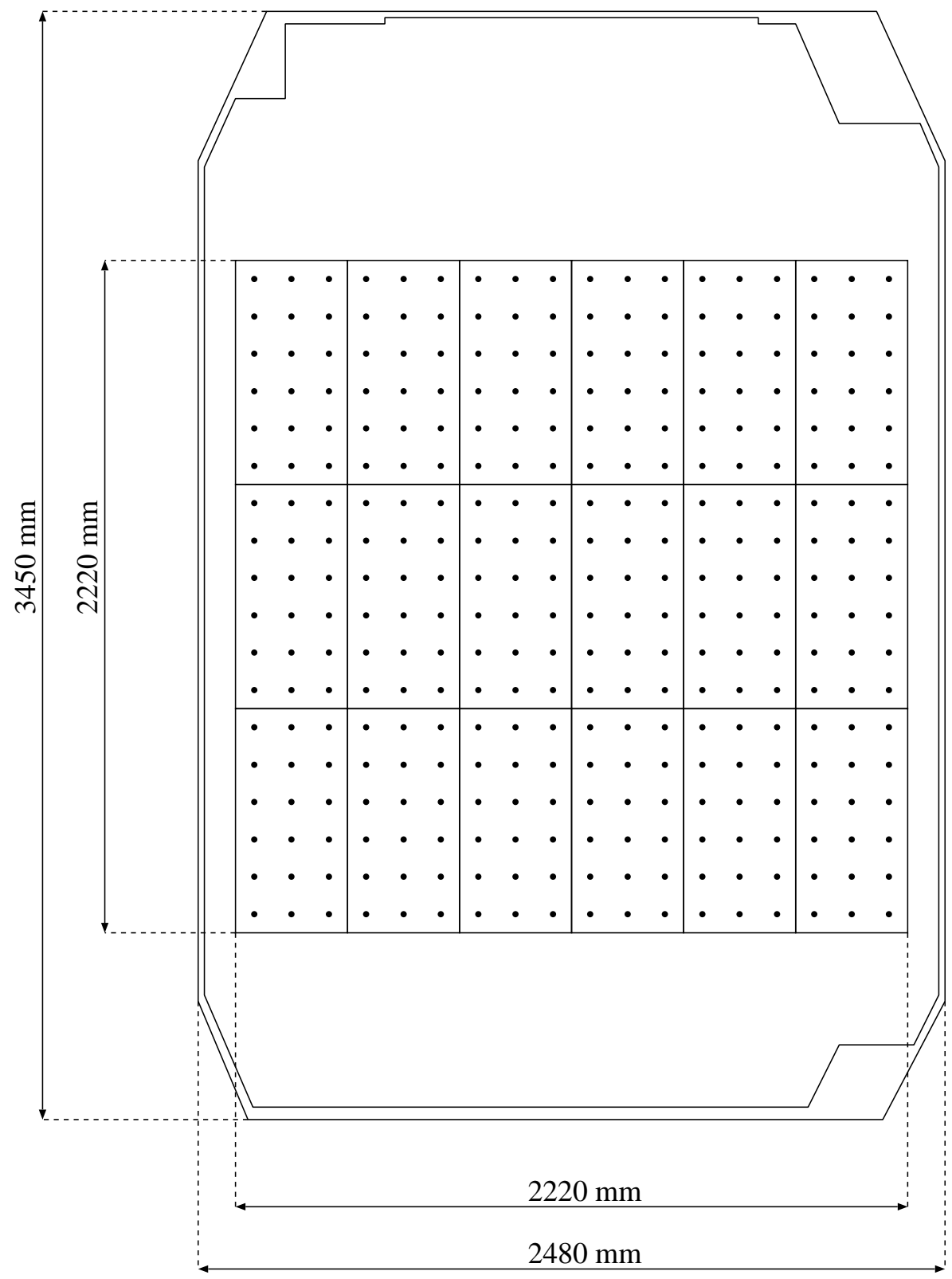

Figure 6: Front view of ET1 and ET2, showing the arrangement of 18 emulsion plates. The position of the X-ray guns embedded in the honeycomb board are shown by black dots. 

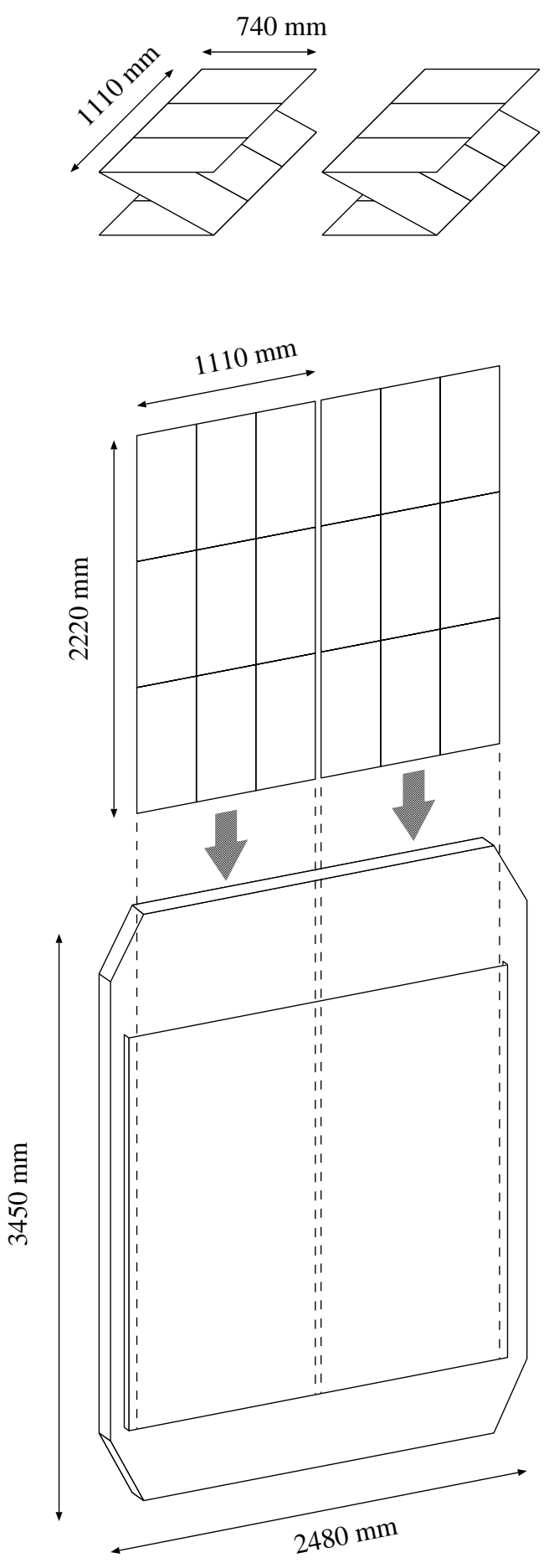

Figure 7: Schematic diagram of the installation of emulsion packages. The unfolded packages are inserted into a plastic film bag on the honeycomb board. Then, the bag is closed and the packages are vacuum-fixed to the board. 


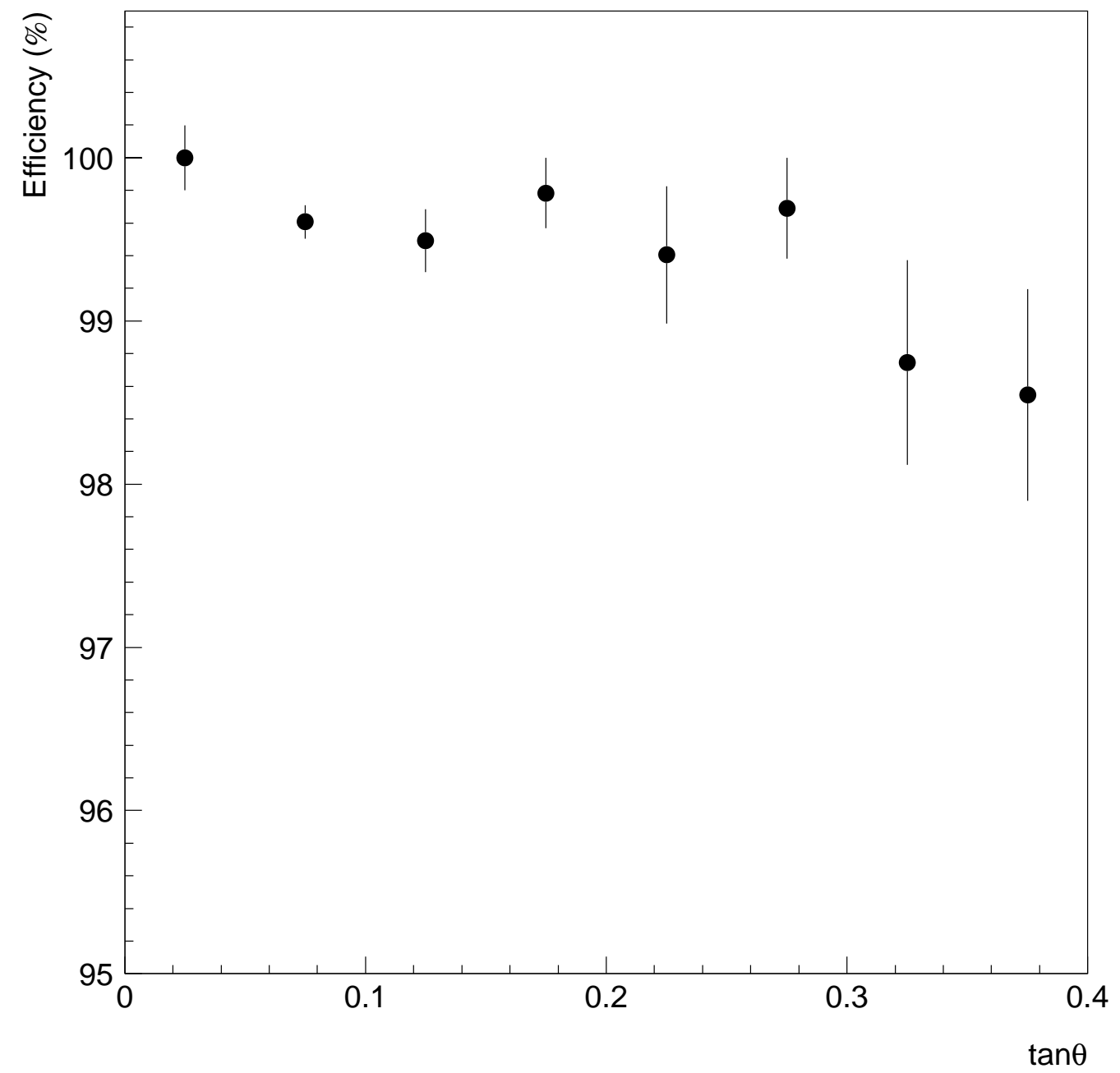

Figure 8: Finding efficiency of the emulsion scanning system. $\theta$ is the angle between the track and the perpendicular to the emulsion sheet. 


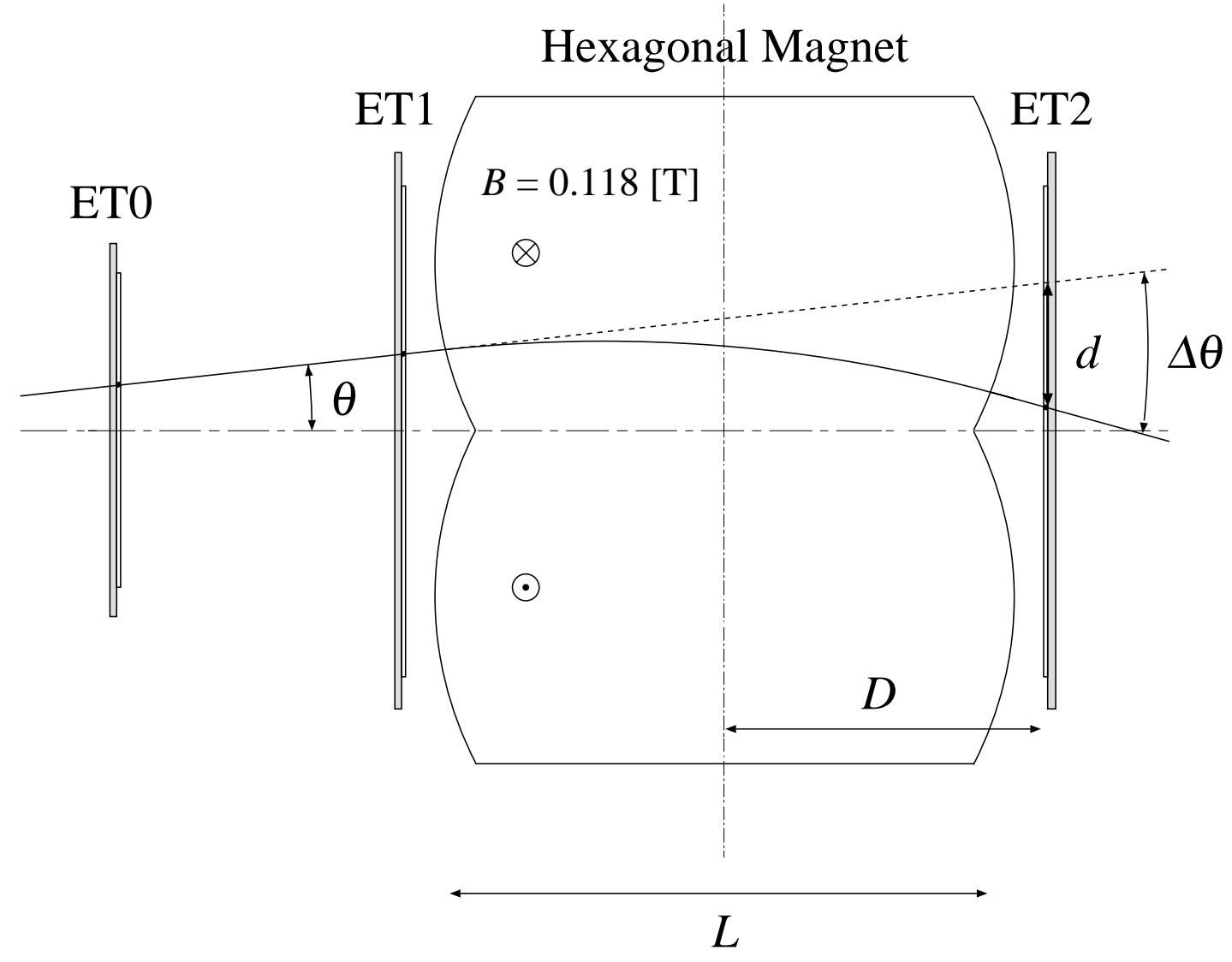

Figure 9: Schematic view of the Hexagonal magnet and ET planes in the bending plane. The solid line shows the track trajectory curved by the magnetic field. The dashed line is the straight line defined by the positions measured in ET0 and ET1. 


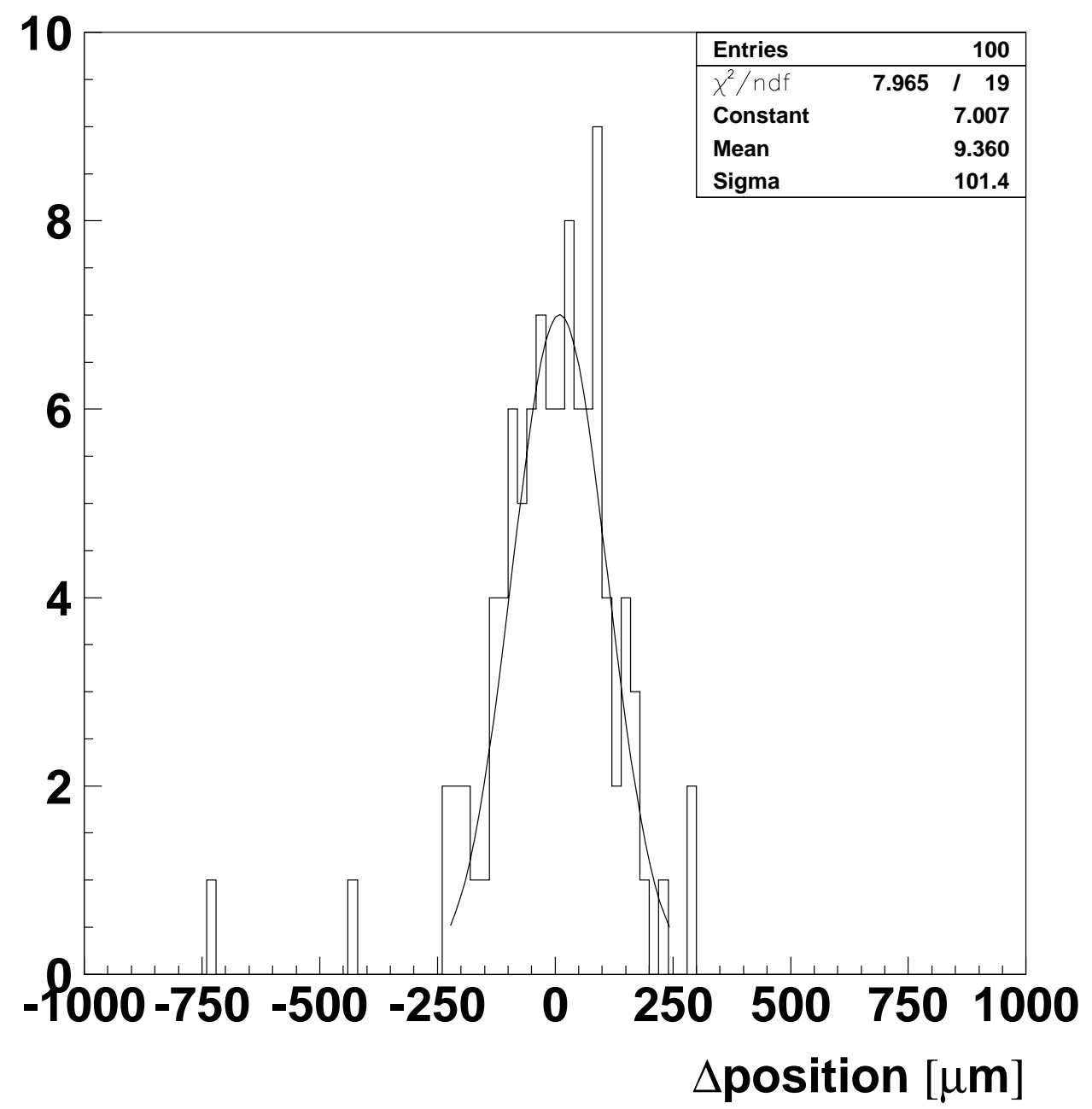

Figure 10: Deviation of tracks found in ET2 after the ET-ET alignment. 


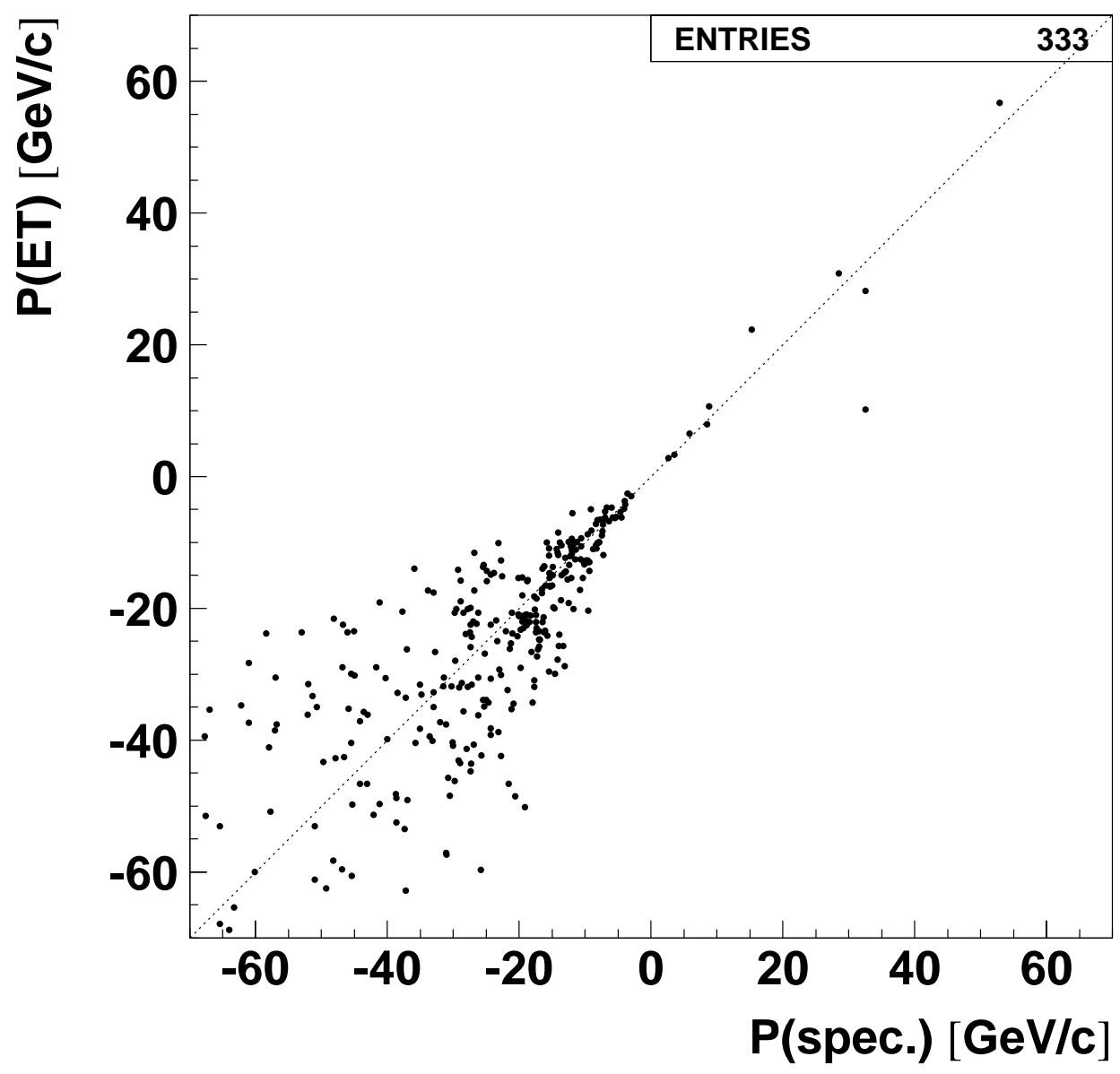

Figure 11: Comparison of the momenta measured in the muon spectrometer and in the ET. 


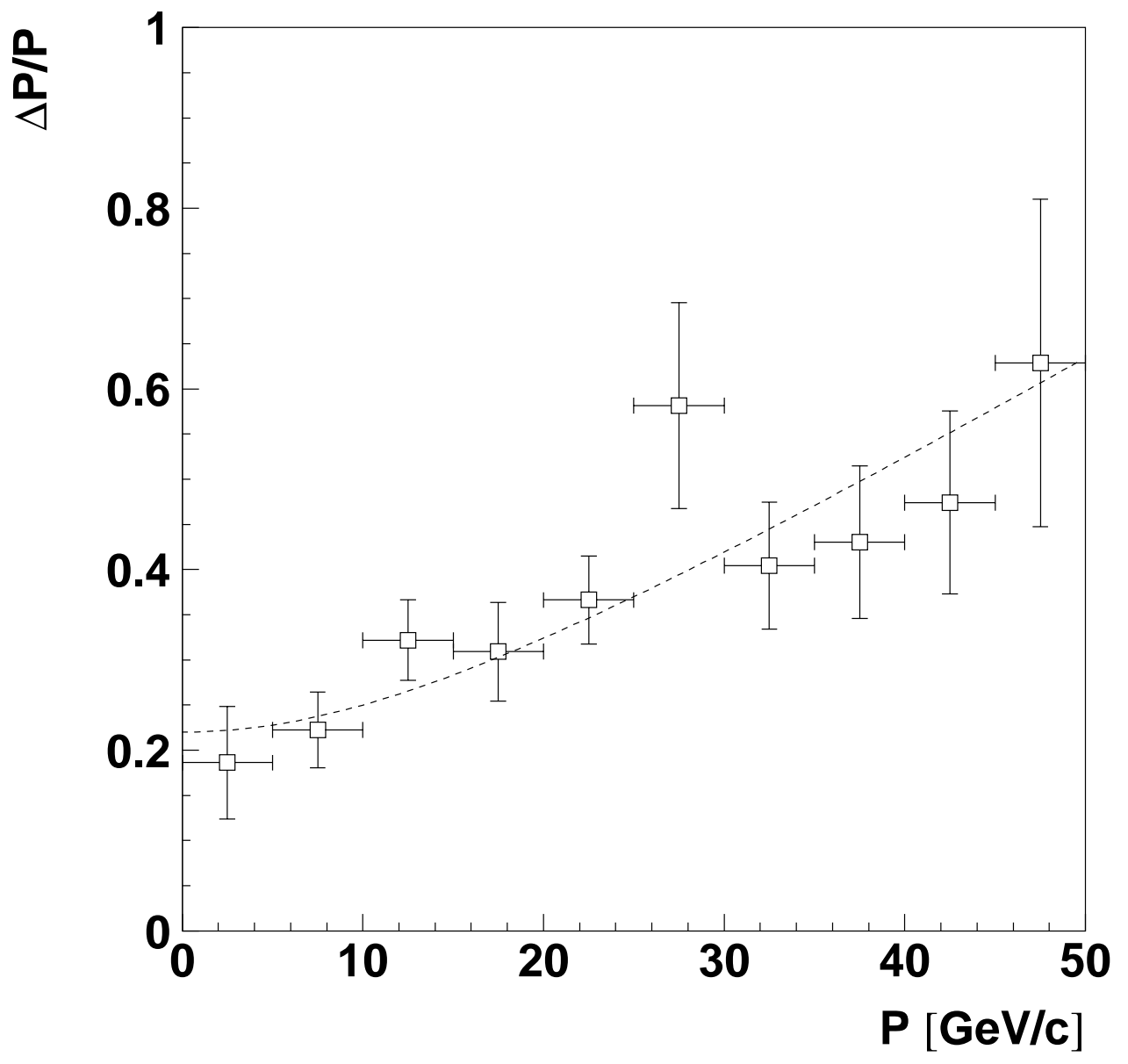

Figure 12: R.M.S. values of the relative difference between the measurements in the muon spectrometer and in the ET as a function of the muon spectrometer momentum. 


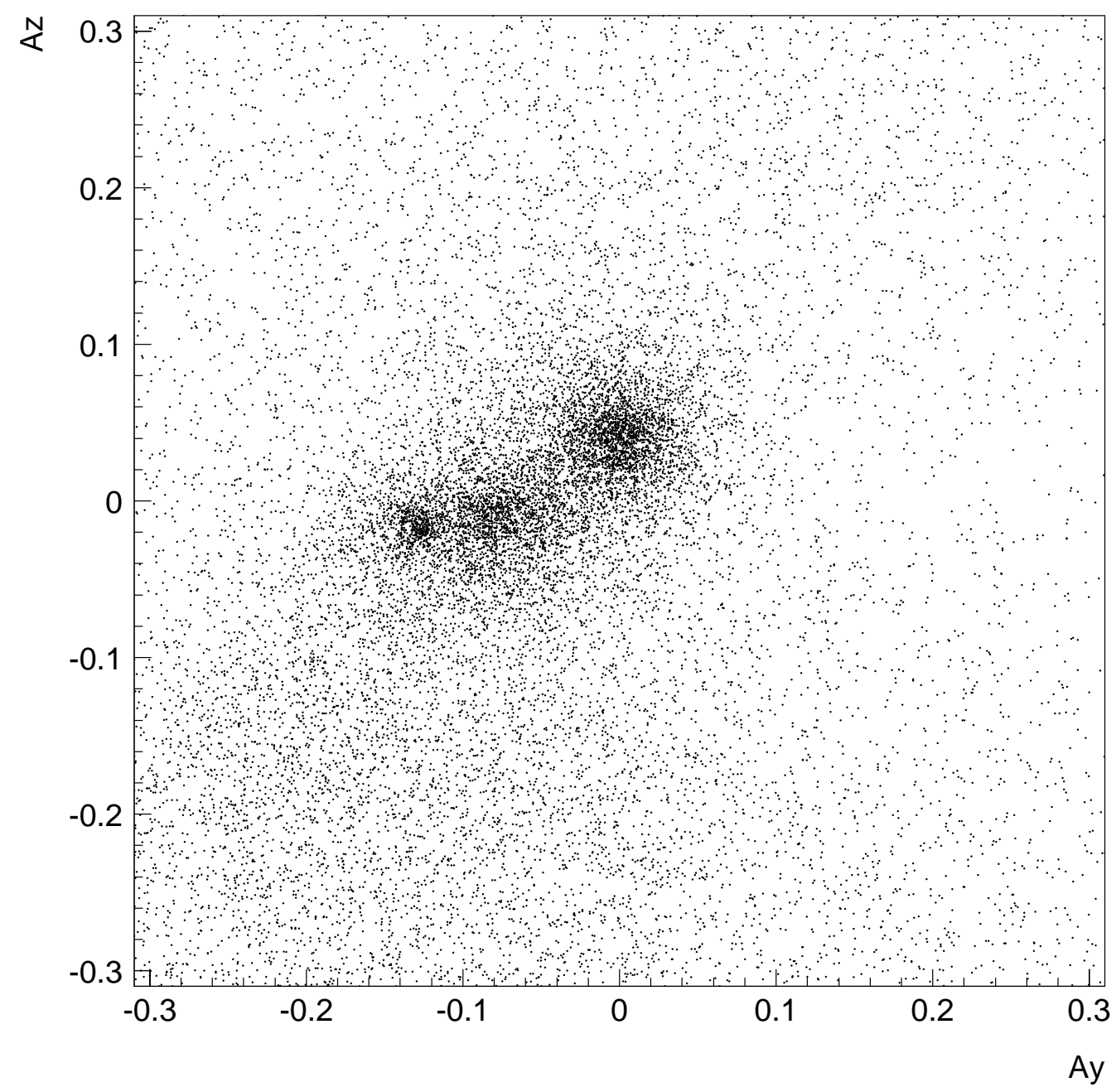

Figure 13: Slope distribution of background tracks. 


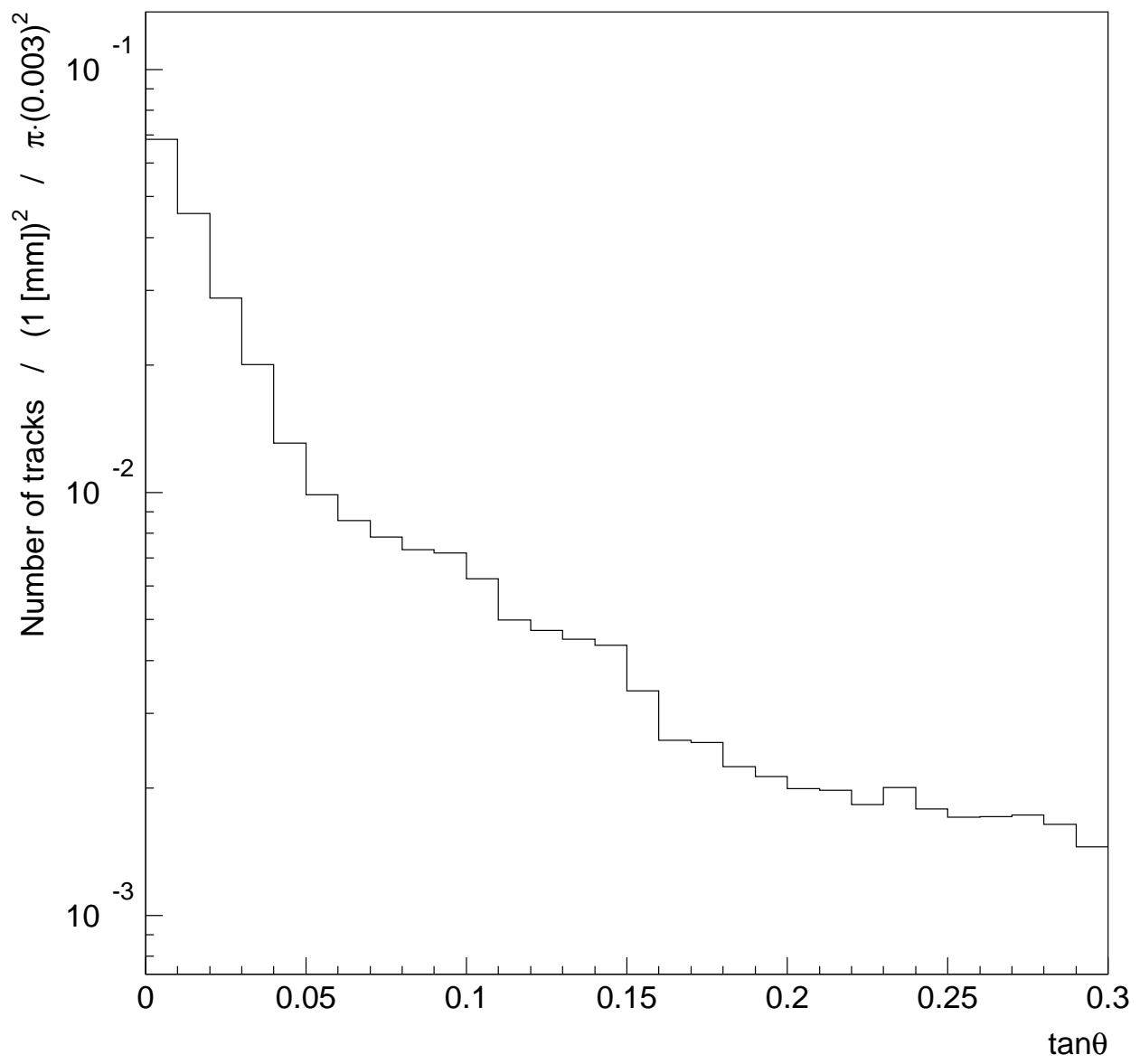

Figure 14: Number of background tracks as a function of the track slope with respect to the neutrino beam direction. 


\begin{tabular}{|c|c|c|c|c|}
\hline & Material & $x[\mathrm{~mm}]$ & $X_{0}[\mathrm{~mm}]$ & $x / X_{0}[\%]$ \\
\hline \multirow{3}{*}{ Hexagonal magnet } & Al-alloy & $2.5 \times 2$ & 89 & 5.62 \\
\cline { 2 - 5 } & Polycarbonate & $2.0 \times 2$ & 346 & 1.16 \\
\hline \multirow{3}{*}{ DT } & Polystyrene scintillator & $3.2 \times 2$ & 424 & 1.51 \\
\cline { 2 - 5 } & NEMA G10 plate & $0.8 \times 4$ & 194 & 1.65 \\
\hline \multirow{2}{*}{ ET } & Emulsion & $0.1 \times 2$ & 28.9 & 0.69 \\
\cline { 2 - 5 } & Plexiglas & 0.8 & 344 & 0.23 \\
\cline { 2 - 5 } & NEMA G10 plate & 0.8 & 194 & 0.41 \\
\hline Insulation curtain & Insulray IR 305-10 & 0.36 & 28.7 & 1.25 \\
\hline \hline \multicolumn{4}{|l|}{ Total } \\
\hline
\end{tabular}

Table 1: Materials in the hadron spectrometer. $x$ and $X_{0}$ denote the thickness along the $X$ axis and the radiation length of each material. 


\begin{tabular}{|c|c|c|c|c|}
\hline & \multicolumn{2}{|c|}{ Magnet off } & \multicolumn{2}{c|}{ Magnet on } \\
\cline { 2 - 5 } & $\sigma_{\text {position }}[\mu \mathrm{m}]$ & $\sigma_{\text {slope }}\left[\times 10^{-3}\right]$ & $\sigma_{\text {position }}[\mu \mathrm{m}]$ & $\sigma_{\text {slope }}\left[\times 10^{-3}\right]$ \\
\hline ET0 & 120 & 1.5 & 160 & 2.2 \\
\hline ET1 & 150 & 1.5 & 220 & 1.8 \\
\hline ET2 & 220 & 1.5 & 250 & 1.8 \\
\hline
\end{tabular}

Table 2: R.M.S. values (position and slope) of the tracks found in the ET planes from the fiber tracker predictions after the ET-DT alignment. 